\title{
18. PHENOMENA OF NON-STABILITY IN CLOSE BINARY SYSTEMS
}

\author{
D. J. MARTYNOV \\ Engelhardt Observatory, Kasan, U.S.S.R.
}

(1) The non-stability of the components in close binary systems has been established in a number of systems, mainly among the eclipsing variables. It cannot be regarded as an unusual phenomenon.

Several types of non-stability may be pointed out:

(a) Physical variability of one or two of the components as, for example, in the following systems:

\begin{tabular}{|c|c|c|}
\hline Star & Spectral types & Remarks \\
\hline S Dor & P Cyg & \\
\hline AR Pav & $\mathrm{W}+\mathrm{P}+\mathrm{cF}$ & \\
\hline VV Cep & $\mathrm{B}_{3}+\mathrm{gM}_{2}$ & \\
\hline $\begin{array}{l}32 \text { Cyg } \\
\text { RX Cas }\end{array}$ & $\begin{array}{l}\mathrm{B} 8+\mathrm{cK}_{5} \\
\mathrm{gA}_{5} \mathrm{e}+\mathrm{gG}_{3}\end{array}$ & $\begin{array}{l}\text { Pulsation is observed with a period } \\
\text { greatly exceeding orbital period }\end{array}$ \\
\hline UX Mon & $\mathrm{A}+\mathrm{Go}-2$ (III-IV) & \\
\hline U Peg & $\mathrm{F}_{3}+\mathrm{F}_{3}$ & $\begin{array}{l}\text { Variations of brightness are particularly } \\
\text { large in ultra-violet }\end{array}$ \\
\hline VW Cep & $\mathrm{dG}_{5}+\mathrm{dK}_{\mathrm{I}}$ & \\
\hline UX UMa & $\mathrm{B}_{3}(\mathrm{sd})$ & Same as U Peg \\
\hline
\end{tabular}

Non-stability of this kind is obviously a feature not confined to binary systems.

(b) The presence of emission bands or lines in the spectrum in every or some phases of the orbital motion as, for example, in the case of the eclipsing variables:

$\begin{array}{ll}\quad \text { Star } & \text { Spectral types } \\ \text { V 444 Cyg } & \text { WN }_{5}+\mathrm{O}^{6} \\ \text { CQ Cep } & \text { WN6 } \\ \text { CX Cep } & \text { WN }_{5} \\ \text { V729 Cyg } & \text { Of }+\mathrm{O}_{9}\end{array}$

The same phenomenon is observed in the case of HD 228786 and some other spectroscopic binaries having Wolf-Rayet components. It is suggested that the absorption spectra of all Wolf-Rayet stars originates in the satellite of type $\mathrm{O}$. There exists a still more radical opinion that all Wolf-Rayet stars are binaries. Thus, non-stability of the Wolf-Rayet type may be typical for close binary systems.

$$
\text { I } 38
$$


Closely similar to these stars are systems in which the intensity of the emission characteristics, if they are observed in all phases of the orbital motion, varies with the phase. Such objects are:

\begin{tabular}{|c|c|c|}
\hline Star & Spectral types & Remarks \\
\hline CQ Cep & WN6 & $\begin{array}{l}\mathrm{He} \text { in } \lambda 4686 \text { is most intense } \\
\text { at conjunctions }\end{array}$ \\
\hline $\begin{array}{l}* \beta \text { Lyr } \\
\text { V } 367 \text { Cyg } \\
\text { RZ Oph } \\
\text { *RX Cas } \\
\text { SX Cas } \\
\text { UX Mon }\end{array}$ & $\begin{array}{l}\mathrm{B} 9+\mathrm{F} \\
\mathrm{B}_{-}-9(?) \text { or } \mathrm{Fo}( \pm 2) \mathrm{III}-\mathrm{v} \\
\mathrm{F}_{3} \mathrm{I}+\mathrm{gK}_{5 \mathrm{p}} \\
\mathrm{gA}_{5} \mathrm{e}+\mathrm{gG} 3 \\
\mathrm{cA} 6+\mathrm{G} 6 \\
\mathrm{~A}+\mathrm{Go}_{0}-\mathrm{G}_{2}(\mathrm{III}-\mathrm{IV})\end{array}$ & Same as CQ Cep \\
\hline
\end{tabular}

These characteristics are manifested by other systems only in certain phases of the orbital cycle as, for instance, in:

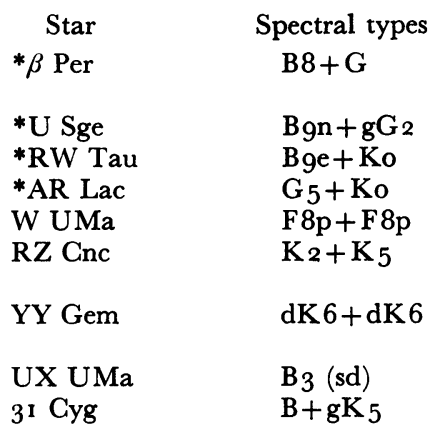

Remarks
The possible ejections observed at the maxi-
mum phase of the eclipse appear only rarely
The same as $\beta$ Per
The same, but more stable
$\mathrm{H}$ and $\mathrm{K}$ are in emission
$\mathrm{H}$ and $\mathrm{K}$ are in emission in the $\mathrm{K}_{2}$
component
$\mathrm{H}, \mathrm{K}$ and hydrogen lines are in emission in
both components
$\mathrm{H} \beta$ is in emission at certain phases
Turbulent motions exist in the atmosphere
of the $\mathrm{K}_{5}$ component

(c) This non-stability manifests itself in sharp differences between the spectroscopic and photometric elements of the system. The discrepancies are caused by gaseous streams in the regions where the absorption lines originate. These lines are either displaced, due to the Doppler effect, or have profiles distorted owing to the same cause. Such phenomena are found in the following systems:

$\begin{array}{ll}\quad \text { Star } & \text { Spectral types } \\ \text { RZ Sct } & \mathrm{B}_{2} \\ \text { *U Cep } & \mathrm{B}^{2}+\mathrm{gG}_{2} \\ \text { *RX Cas } & \text { gA } 5 \mathrm{e}+\mathrm{gG}_{3} \\ \text { SX Cas } & \text { cA6+G6 } \\ \text { UX Mon } & \mathrm{A}+\mathrm{Go}-\mathrm{G}_{2} \text { (III-IV) }\end{array}$

(d) This non-stability manifests itself in an unsteadiness of the period of orbital motion. In some cases the observed times of light minima cannot be represented either by a linear ephemeris or by any other formula, if the latter is applied for an interval of time different from that for which it was deduced. Such non-stability is represented by a number of systems not 
enumerated here. It should, however, be pointed out that a number of the systems already mentioned have inconstant photometric periods (such systems are marked with an asterisk).

(2) As is well known, stars of quite different physical properties (revealed by their spectra and absolute magnitudes) are found in close pairs. Certain combinations are never met with as, for example, a giant together with a dwarf of late spectral type. This might easily be understood as a result of observational selection. Therefore, the non-stability of type $(a)$ is actually not typical for binary systems. Contrariwise, the origin of the non-stability of type $(b)$ is facilitated by the existence of a companion near a given star, or is caused by the tidal action of the secondary. In the above lists we meet representatives of about all types of stars from the spectrum-luminosity diagram. This means that normal and hot giants, B- and A-type stars, ordinary dwarfs, sub-dwarfs and sub-giants are found among unstable stars. The features of non-stability (the presence of a gaseous ring) are so weak in the systems of U Sge, RW Tau and possibly $\beta$ Per, that they become visible only when the total, or nearly total, eclipses cut off the light of the photosphere of the bright component. If no eclipses should occur, we would know nothing regarding such types of non-stability, especially since they are transient phenomena. At the same time these phenomena are apparently confined to close binaries. They may exist in a number of spectroscopic binaries, but remain unobserved in the absence of eclipses.

The eclipsing variable DQ Her-a former nova-is an example of another form of non-stability. This non-stability was, perhaps, the cause of the origin of this extremely close binary system, whose spectrum even now manifests features of non-stability. We do not know, however, how long such features will survive.

(3) We have at present for the explanation of the non-stability in close binary systems the so-called Kuiper-Struve mechanism. At a sufficiently small separation of the components, the geometrical dimensions of one or both may surpass the limits of the internal or external equipotential surfaces. There will take place in such a case either the exchange of matter between the components along the equipotential surfaces, or an ejection of matter from the system through the second Lagrangian point, on the condition that the total energy of the particle will exceed some critical value $h_{L_{2}}$. If neither of the components of such a 'contact binary star' surpasses the limits of the outer equipotential surface, ejection of matter might, however, take place owing to the thermal non-stability of one component. That is, macroscopic motions in the stellar atmosphere might lead to an 
ejection of matter possessing sufficient kinetic energy beyond the limits of the internal equipotential surface. In this case the phenomenon, which would have no consequences for a single star, would lead in a double system to a loss of mass and of rotational momentum. Spectroscopic effects of such ejection have been discovered by O. Struve and others since $194 \mathrm{I}$ in a number of photometric binary systems.

As concerns photometric effects, the asymmetry of light curves observed in some eclipsing variables might be explained, as it was first by Mergentaler in 1950, in terms of streams of gaseous material. Indeed, large masses of cool gas actually cover the photosphere of the star, decreasing its surface brightness. In so far as the gaseous stream is asymmetric, the steepness of the light curve and the total brightness of the system before the eclipse and after will be quite different.

Unfortunately, the quantitative deductions of these effects made by Mergentaler, Dadaev, Sofronicky, and Svetchnikov were carried out by these authors under extremely simplified conditions, because of difficulties of a mathematical nature. These calculations show only that the expected effects will be of the same order as the ones observed. A more detailed mathematical and physical analysis of this phenomenon is extremely desirable for the elimination of the effects of asymmetry in the observed light curves. All the modern methods for the solution of the light curves of eclipsing variables have attained a high degree of perfection, but without taking into account this asymmetry.

Svetchnikov calculated also the intensity of the emission lines that might be observed in the spectrum of the star in the presence of gas streams, and found them (also under simplified assumptions) to correspond with the observed intensity of the emission lines.

All these results render the hypothesis of the ejection from the contact binaries trustworthy, but not yet proven by far.

(4) However, the hypothesis of ejection is supported in another way, since it may be used for the explanation of irregular changes in the length of the orbital period of a binary system. As has been said before, such variations of the period are a common phenomenon in eclipsing variables.

Celestial mechanics points out only two sources of the variation of the photometric period of a binary system: motion around a third body and motion of the apsidial line in the case of eccentric orbit. These two cases are represented in the eclipsing variables by some reliable examples.

The motion around a third body is observed in the cases of $\beta$ Per, RT Per(?), and possibly SW Cyg; the motion of the apsidal line is observed in the binary systems YY Cyg, RU Mon, GL Car, V526 Sgr, and YY Sgr. 
Both motion of the apsidal line and motion around a third body, the theories of which give a number of additional terms in the expression for the epochs of photometric conjunctions, are manifested by periodic (usually long-periodic) terms in the epochs of minima. Meanwhile, irregular variations of the epochs of minima of eclipsing variables, however, are quite beyond doubt in a number of cases. The circumstance that the photometric period does not coincide with the sidereal period of orbital motion cannot explain this phenomenon, because the observed differences even in the case of the libration of ellipsoidal components (if librations are possible in a system of gaseous stars), must also be regular and not erratic.

It is clear that the fluctuations of the photometric period in a binary system correspond to the actual fluctuations of the sidereal period of orbital motion, which is possible only if changes of the rotational momentum of the system are taking place. The hypothesis of gaseous streams makes such fluctuations admissible. Individual spontaneous fluctuations of the period might be explained, as was done by Wood, by transitory ejections of very large masses of matter from a star and a system. Whereas a continuous loss of mass by a system must lead to a secular increase of the period corresponding to the law $a\left(\mathbf{m}_{1}+\mathbf{m}_{2}\right)=$ constant, a one-sided ejection (depending upon its direction) may cause the period to become longer or shorter. As is seen from the calculations by Wood and by Svetchnikov, the observed fluctuations of the period in RZ Cas, U Cep, AO Gas, AR Lac and U Sge require an ejection of the order of $10^{-7}$ or $1^{10^{-6}}$ solar masses, If we remember that the masses ejected at nova outbursts exceed the aforementioned values only by one order of magnitude, it seems doubtful that ejections of masses of the order of $10^{-7}$ or $10^{-6}$ solar masses might pass unnoticed for stars under frequent observation. But it must also be remembered that the above ejections will explain the observed spontaneous changes in the periods, if they represent a series of similarly directed ejections that continue for a considerable interval of time, for example, a year. The photometric effects calculated by Svetchnikov (though under simplified conditions) will then be found to be sufficiently weak. However, single large changes of the period require the simultaneous ejection of large masses, which could not escape the attention of the observers.

As was shown by the author some time ago, the phenomenon of tidal friction might, under certain conditions, facilitate the mutual approach of the components in binary systems, if the viscosity of matter and of radiation are considerable in a star. Ejections of matter, if directed in a suitable way, might assist this approach. Thus, the components may approach each other so closely that the ejection of matter from them will become extremely 
intense. But a similar stage of strong non-stability cannot be of long duration, because the law $a\left(\mathbf{m}_{1}+\mathbf{m}_{2}\right)=$ constant will lead to an increase of the distance between the components. If tidal friction is absent, the stage of non-stability of a star will be of very long duration (of the order of millions of years) only if the change of the structure of a component of the system will lead to a progressive increase in its dimensions.

Thus we find a number of mechanisms that make the components of close binaries non-stable in the course of considerable intervals of time. This makes their evolution quite different from the evolution of a single star.

\section{BIBLIOGRAPHY}

A. N. Dadaev, Pulkovo Bull. 19, No. 5, 31 (1954).

Z. Kopal, Jodrell Bank Ann. 1, 37 (1954).

G. P. Kuiper, Ap.7. 93, I 33 (1941).

D. J. Martynov, Publ. Engelhardt Obs. No. 25 (1948).

J. Mergentaler, Contr. Wroclaw Astr. Obs. No. 4 ( (1950).

A. V. Sofronicky, Pulkovo Bull. 19, No. 4, I (1953).

A. V. Svetchnikov, Variable Stars, 10, 262 (1955).

F. B. Wood, $A p$. . . 112, 196 (1950). 